\title{
An Experimental Investigation on Vegetable Oils as a Cutting Fluid under Minimum Quantity Lubrication: A Sustainable Machining Approach
}

\author{
Shrikant U. Gunjal ${ }^{*}$,Sudarshan B. Sanap ${ }^{2}$, Nilesh C. Ghuge ${ }^{3}$, Satish Chinchanikar ${ }^{4}$ \\ ${ }^{1 *}$ Research Scholar, MIT Art, Design and Technology University, Pune, India \\ ${ }^{2}$ Professor, MIT Art, Design and Technology University, Pune, India \\ ${ }^{3}$ Professor, Matoshri College of Engineering and Research Center, Nashik, India \\ ${ }^{4}$ Profesor, Vishwakarma Institute of Information Technology, Pune, India \\ shrikant.qunjal@mituniversity.edu.in
}

\begin{abstract}
Cutting fluid is a vital part of the machining process. Cutting fluid is significantly applied to lower the friction and heat generated in the machining zone. It also helps in easy chip removal, protection against oxidation, tool life improvement, and an overall improvement in the quality of the product. The current industrial practices are majorly emphasized on mineral-based oil application under flood lubrication to achieve superior quality. However, these oils and techniques are toxic and environmentally unfriendly. Machining under dry or with minimum quantity lubrication (MQL) has been mostly preferred to eliminate the use of abundant oil. The current research work has established the promising potential for vegetable oils as a cutting fluid under MQL during turning of AISI 4130 steel. The results inferred that vegetable-based cutting fluids performed better over mineral-based cutting fluids in terms of lower values of machined surface roughness, tool wear, cutting forces, and chip-tool interface temperature. The MQL machining performance in terms of cutting forces, surface roughness and tool life has been observed better in comparison to machining under flood and dry cutting conditions.
\end{abstract}

Keywords:Turning, Cutting parameters, MQL, Vegetable Oils, Cutting force, Surface roughness

\section{Introduction}

Manufacturing industries are striving to achieve superior product quality with minimumresource utilization. Aiming for higher productivity is closely associated with higher material removal rate which results in extreme heat generation. The heat generated at the machining zone leads to an adverse effect on the machining process in terms of uneven surface quality and abrupt tool failure in certain cases. Cutting fluids are the source of limiting the heat generation during machining attributed to their cooling and lubrication characteristics. However, the current practices of mineral-based cutting fluids and flood lubrication technique resulting in a threat to the environment and operational safety, as the exposure of mineral-based cutting fluids for a longer time could result in dermatological and respiratory issues even [1, 2]. Concerning the same, many environmental protection agencies (EPA) have insisted long back to eliminate the application of mineral-based cutting fluids projecting their carcinogenic nature subsequent issues like disposal and recycling [3-5]. The rising ecological threat concerning the mineral-based cutting fluids and flood lubrication technique enforce to find the alternative for sustainable development. Dry machining was potentially projected as the method to eliminate the use of cutting fluids leading to a green manufacturing approach therein [6]. However, the certain limitations of dry machining were exposed especially at higher cutting speed as increased wear rate was noted as the major concern [7]. Thus, the industries are looking forward to environment-friendly machining which would have a long-term sustainable approach and a potential alternative to flood and dry technique. Near-dry machining which is also termed as minimum quantity lubrication or micro lubrication emerges in the same regard [8].

Minimum quantity lubrication (MQL) uses a cutting fluid in the range of 50-150 $\mathrm{ml} / \mathrm{hr}$ which is significantly low as compared to the flood lubrication system (1-10 ltr/min), so it is appropriately termed as micro lubrication or near-dry machining [9]. The mixture of compressed air and cutting fluids is applied at the machining zone through the nozzle for precise penetration. The minimum attributed cutting oil usage avoids the 
wastage, saves the cost regarded to it, and reduces the chances of direct cutting exposure of cutting fluid to operator significantly [10-12]. Varadarajanet. al. 2002 [13], noted the superior performance of MQL over the dry and wet method during the hard turning. Results inferred the improved tool life and surface finish. Reduction in cutting temperature under the application of MQL attributed to increasing fluid flow rate and air pressure; was observed by Juet. al, 2005 [14]. Attanasioet. al, 2006 [15] noted the improved tool life under the application of MQL over the dry machining due to reduced flank wear. Liao et. al. 2007 [16], observed the compatibility of the MQL application at high-speed milling over the dry and flood lubrication as the tool life was noted more under it. Better surface integrity was noted under the optimized cutting conditions for MQL during the grinding process [17]. Hadad and Sadeghi, 2013 [18]analyzed the comparative performance of MQL, dry, and flood techniques during the turning of AISI 4140 steel. They noted the least value of cutting forces under the application of MQL. Kedareet. al., 2014 [19] evaluated the compatibility of MQL at varying cutting conditions during the milling operation. The comparative study of MQL and flood lubrication revealed the improved surface finish by $27 \%$ under MQL by the virtue of reduced cutting temperature.

Cutting fluids being the media to lower the heat generation at the cutting zone and reduction in its subsequent effects; has been widely used to achieve superior product quality in all aspects. Considering the environment-friendly approach for sustainable machining practices, vegetable-based cutting fluids are the best source of cooling and lubrication under the application of MQL. Vegetable-based cutting fluids which are also called as biolubricants are the plant-based agricultural products possess high flash point, viscosity, and boiling point; attributed to their triglyceride structure. Vegetable-based cutting fluids are compatible with high-temperature applications as well attributed to their high flash point and strong and homogenous molecular film. It significantly reduces the fire hazard and smoke formation hazard [20, 21]. Khan and Dhar, 2006 [22] have noted the significant reduction in tool wear along-with the improved dimensional accuracy and surface finish under the application of vegetable oil-based cutting fluids using MQL. Islam and Dhar, 2008 [23] found a reduction in surface roughness and grinding zone temperature using vegetable oils during the grinding operation of AISI 1060 steel material. Comparative evaluation of biolubricants performed by Gunjal and Patil, 2018 [24] inferred that the canola oil provides improved surface finish during hard turning of AISI 4340 material. Application of biolubricants under MQL even reduces the traces of built-up edge formation. The chip thickness study performed by Gunjal et. al., 2020 [25] reveals the lower energy consumption during hard turning operation by the virtue of maximum shear angle attributed to minimum chip thickness. In continuation of their work, Gunjal and Sanap, 2020 [26] have noted the improved tool life under the MQL application. Many researchers [27-30] with their experimental investigations and relevant studies on different alloys and hard-to-cut materials under varying cutting conditions and different machining operations have noted the better output under MQL in terms of reduced tool wear, surface roughness, cutting temperature, cutting forces along-with improved tool life and surface integrity. MQL overall is proven to be the most sustainable approach [31].

\section{Experimental work}

Experimental investigations are undertaken for Turning of AISI 4130 steel material using vegetable-based cutting fluids. Three different machining environments; MQL, flood, and dry are compared thoroughly. Following table 1 shows the cutting conditions and relevant details under consideration. A total of 27 experiments were performed varying the cutting speed, feed, and depth of cut at different levels as depicted in Table 1.

Table1.Experimental Conditions

\begin{tabular}{|c|c|c|}
\hline $\begin{array}{c}\text { Sr. } \\
\text { No. }\end{array}$ & Parameter & Description \\
\hline 1 & Material & AISI 4130 \\
\hline 2 & Machine & Medium duty lathe \\
\hline 3 & Cutting Tool & Uncoated brazed carbide tool \\
\hline
\end{tabular}




\begin{tabular}{|c|c|c|}
\hline 4 & $\begin{array}{c}\text { Machining } \\
\text { Environment }\end{array}$ & MQL, Flood, Dry \\
\hline 5 & Cutting Fluids & $\begin{array}{c}\text { Sunflower, Soybean, Groundnut, } \\
\text { Coconut, Blasocut 4000 }\end{array}$ \\
\hline 6 & $\begin{array}{c}\text { Cutting speed (Vc) } \\
\text { (m/min) }\end{array}$ & $34.27,53$, and 79.73 \\
\hline 7 & Feed (f) (mm/rev) & $0.35,0.4$, and 0.45 \\
\hline 8 & $\begin{array}{c}\text { Depth of cut (d) } \\
\text { (mm) }\end{array}$ & $0.5,1$, and 1.5 \\
\hline
\end{tabular}

\subsection{Experimental setup and design of experiment}

For the present experimental investigations, the medium-duty lathe machine and MQL system are used as shown in figure 1.

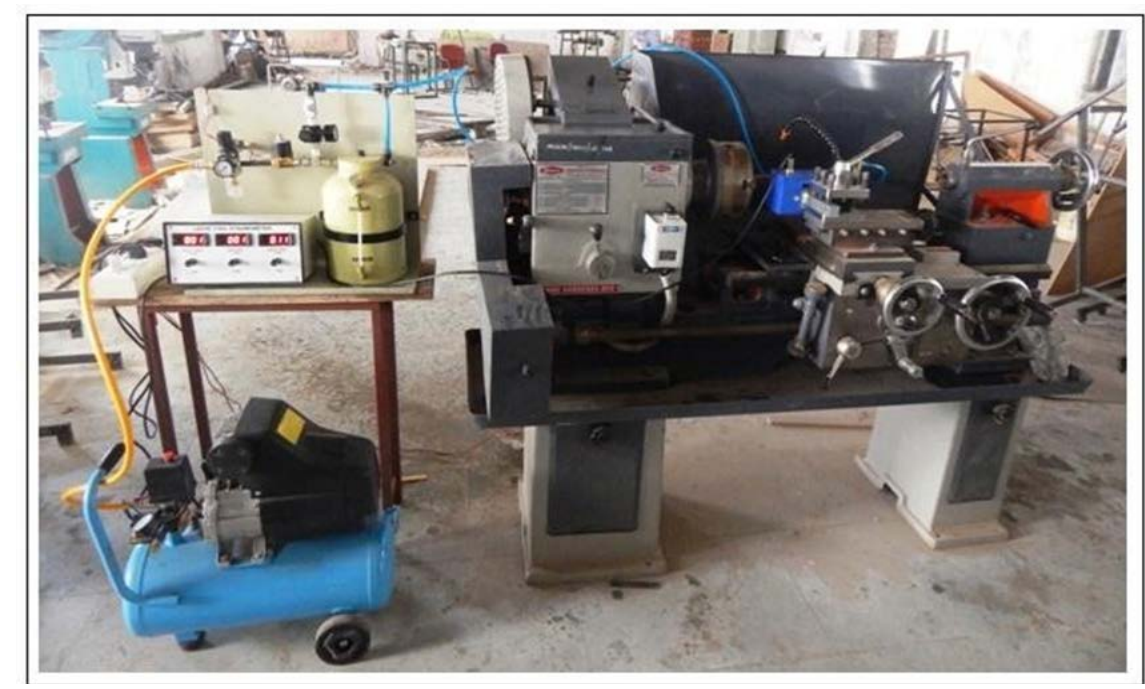

Figure1.The photographic view of the experimental set-up

\section{Results and discussion}

This section describes the measuring parameters for the current experimental investigations. Cutting force $(\mathrm{Fc})$, surface roughness $(\mathrm{Ra})$, temperature $(\mathrm{T})$, and power consumption $(\mathrm{P})$ are measured for the different machining environments under the varying cutting fluid application. The lathe tool dynamometer is used to measure the cutting forces, while surface roughness and the temperature are measured with the portable roughness tester and infrared thermometer respectively. Three major cutting environments viz. dry, flood, MQL are used for the performance evaluation. Following tables, 2-5 show the observations concerning the design of the experiment under each cutting condition with different oils under consideration.

Table2.Observations under Dry Environment

\begin{tabular}{|c|c|c|c|c|c|c|c|}
\hline \multirow{2}{*}{$\begin{array}{c}\text { Run } \\
\text { No. }\end{array}$} & $\mathrm{Vc}$ & $\mathrm{f}$ & $\mathrm{d}$ & $\mathrm{Fc}$ & $\mathrm{Ra}$ & $\mathrm{T}$ & $\mathrm{P}$ \\
\cline { 2 - 8 } & $\mathrm{m} / \mathrm{min}$ & $\mathrm{mm} / \mathrm{rev}$ & $\mathrm{mm}$ & $\mathrm{N}$ & $\mu \mathrm{m}$ & ${ }^{\circ} \mathrm{C}$ & $\mathrm{KW}$ \\
\hline 1 & 34.27 & 0.35 & 0.5 & 382.95 & 3.70 & 82.95 & 0.22 \\
\hline 2 & 34.27 & 0.35 & 1 & 444.96 & 3.95 & 94.78 & 0.26 \\
\hline 3 & 34.27 & 0.35 & 1.5 & 491.17 & 4.02 & 105.61 & 0.28 \\
\hline 4 & 34.27 & 0.4 & 0.5 & 371.43 & 4.03 & 97.61 & 0.21 \\
\hline 5 & 34.27 & 0.4 & 1 & 433.21 & 4.30 & 108.96 & 0.24 \\
\hline 6 & 34.27 & 0.4 & 1.5 & 479.20 & 4.40 & 119.31 & 0.27 \\
\hline 7 & 34.27 & 0.45 & 0.5 & 383.17 & 4.70 & 111.62 & 0.22 \\
\hline
\end{tabular}




\begin{tabular}{|c|c|c|c|c|c|c|c|}
8 & 34.27 & 0.45 & 1 & 444.73 & 4.99 & 122.48 & 0.26 \\
\hline 9 & 34.27 & 0.45 & 1.5 & 490.48 & 5.12 & 132.35 & 0.28 \\
\hline 10 & 53 & 0.35 & 0.5 & 367.90 & 3.49 & 78.95 & 0.33 \\
\hline 11 & 53 & 0.35 & 1 & 430.26 & 3.76 & 93.60 & 0.38 \\
\hline 12 & 53 & 0.35 & 1.5 & 476.81 & 3.86 & 107.24 & 0.42 \\
\hline 13 & 53 & 0.4 & 0.5 & 360.78 & 3.92 & 91.04 & 0.32 \\
\hline 14 & 53 & 0.4 & 1 & 422.91 & 4.21 & 105.21 & 0.37 \\
\hline 15 & 53 & 0.4 & 1.5 & 469.24 & 4.33 & 118.37 & 0.41 \\
\hline 16 & 53 & 0.45 & 0.5 & 376.92 & 4.69 & 102.47 & 0.33 \\
\hline 17 & 53 & 0.45 & 1 & 438.83 & 5.01 & 116.15 & 0.39 \\
\hline 18 & 53 & 0.45 & 1.5 & 484.93 & 5.15 & 128.83 & 0.43 \\
\hline 19 & 79.73 & 0.35 & 0.5 & 358.44 & 3.12 & 76.63 & 0.48 \\
\hline 20 & 79.73 & 0.35 & 1 & 421.29 & 3.42 & 95.30 & 0.56 \\
\hline 21 & 79.73 & 0.35 & 1.5 & 468.34 & 3.56 & 112.97 & 0.62 \\
\hline 22 & 79.73 & 0.4 & 0.5 & 357.61 & 3.69 & 85.05 & 0.48 \\
\hline 23 & 79.73 & 0.4 & 1 & 420.23 & 4.02 & 103.24 & 0.56 \\
\hline 24 & 79.73 & 0.4 & 1.5 & 467.06 & 4.17 & 120.42 & 0.62 \\
\hline 25 & 79.73 & 0.45 & 0.5 & 380.03 & 4.60 & 92.80 & 0.50 \\
\hline 26 & 79.73 & 0.45 & 1 & 442.43 & 4.95 & 110.51 & 0.58 \\
\hline 27 & 79.73 & 0.45 & 1.5 & 489.03 & 5.13 & 127.21 & 0.65 \\
\hline
\end{tabular}

Table3.Observations under the Application of Blasocut Oil (Flood and MQL Environment)

\begin{tabular}{|c|c|c|c|c|c|c|c|c|}
\hline \multirow{3}{*}{$\begin{array}{l}\text { Run } \\
\text { No. }\end{array}$} & \multicolumn{4}{|c|}{ Flood-blasocut } & \multicolumn{4}{|c|}{ MQL-blasocut } \\
\hline & Fc & $\mathrm{Ra}$ & $\mathrm{T}$ & $\mathrm{P}$ & Fc & $\mathrm{Ra}$ & $\mathrm{T}$ & $\mathrm{P}$ \\
\hline & $\mathrm{N}$ & $\mu \mathrm{m}$ & ${ }^{\circ} \mathrm{C}$ & KW & $\mathrm{N}$ & $\mu \mathrm{m}$ & ${ }^{\circ} \mathrm{C}$ & KW \\
\hline 1 & 355.21 & 3.55 & 67.28 & 0.21 & 334.73 & 2.72 & 64.58 & 0.20 \\
\hline 2 & 416.71 & 3.60 & 78.39 & 0.24 & 396.18 & 2.92 & 75.60 & 0.23 \\
\hline 3 & 463.60 & 3.69 & 87.25 & 0.27 & 441.73 & 3.07 & 84.55 & 0.26 \\
\hline 4 & 346.19 & 3.70 & 81.26 & 0.19 & 326.06 & 3.09 & 79.86 & 0.18 \\
\hline 5 & 406.99 & 3.78 & 92.44 & 0.23 & 387.66 & 3.27 & 90.54 & 0.22 \\
\hline 6 & 453.18 & 3.89 & 101.37 & 0.26 & 433.36 & 3.41 & 99.15 & 0.25 \\
\hline 7 & 360.15 & 3.91 & 93.26 & 0.21 & 339.38 & 3.48 & 92.90 & 0.20 \\
\hline 8 & 420.24 & 4.00 & 104.51 & 0.24 & 401.13 & 3.65 & 103.24 & 0.24 \\
\hline 9 & 465.74 & 4.13 & 113.51 & 0.27 & 446.98 & 3.78 & 111.51 & 0.26 \\
\hline 10 & 342.71 & 2.15 & 62.47 & 0.30 & 322.16 & 1.87 & 60.11 & 0.29 \\
\hline 11 & 404.45 & 2.20 & 77.27 & 0.36 & 383.97 & 2.04 & 74.96 & 0.34 \\
\hline 12 & 451.59 & 2.28 & 89.83 & 0.40 & 429.87 & 2.18 & 87.73 & 0.38 \\
\hline 13 & 337.72 & 2.42 & 74.46 & 0.30 & 317.37 & 2.22 & 72.82 & 0.28 \\
\hline 14 & 398.76 & 2.48 & 89.33 & 0.35 & 379.32 & 2.39 & 87.33 & 0.34 \\
\hline 15 & 445.20 & 2.59 & 101.96 & 0.39 & 425.38 & 2.50 & 99.77 & 0.38 \\
\hline 16 & 355.70 & 2.73 & 84.48 & 0.32 & 334.56 & 2.60 & 83.30 & 0.30 \\
\hline 17 & 416.04 & 2.81 & 99.42 & 0.37 & 396.67 & 2.75 & 97.46 & 0.36 \\
\hline 18 & 461.78 & 2.94 & 112.11 & 0.41 & 442.87 & 2.86 & 109.56 & 0.40 \\
\hline 19 & 334.55 & 1.83 & 59.09 & 0.45 & 314.26 & 1.73 & 57.25 & 0.43 \\
\hline
\end{tabular}




\begin{tabular}{|l|l|l|l|l|l|l|l|l|}
20 & 396.63 & 1.87 & 79.16 & 0.52 & 376.57 & 1.87 & 77.56 & 0.50 \\
\hline 21 & 444.12 & 1.94 & 96.99 & 0.59 & 422.99 & 1.97 & 95.80 & 0.57 \\
\hline 22 & 335.30 & 2.25 & 68.25 & 0.45 & 315.00 & 2.07 & 66.31 & 0.43 \\
\hline 23 & 396.69 & 2.31 & 88.39 & 0.53 & 377.46 & 2.20 & 86.28 & 0.51 \\
\hline 24 & 443.47 & 2.40 & 106.28 & 0.59 & 424.03 & 2.28 & 104.18 & 0.57 \\
\hline 25 & 359.03 & 2.72 & 75.44 & 0.48 & 337.72 & 2.43 & 73.12 & 0.45 \\
\hline 26 & 419.72 & 2.79 & 95.64 & 0.55 & 400.34 & 2.55 & 92.75 & 0.53 \\
\hline 27 & 465.80 & 2.91 & 113.60 & 0.62 & 447.05 & 2.62 & 110.31 & 0.60 \\
\hline
\end{tabular}

Table 4.Observations under the Application of Soybean and Sunflower Oil (MQL Environment)

\begin{tabular}{|c|c|c|c|c|c|c|c|c|}
\hline \multirow{3}{*}{$\begin{array}{l}\text { Run } \\
\text { No. }\end{array}$} & \multicolumn{4}{|c|}{ MQL-soybean } & \multicolumn{4}{|c|}{ MQL-sunflower } \\
\hline & Fc & $\mathrm{Ra}$ & $\mathrm{T}$ & $\mathrm{P}$ & Fc & $\mathrm{Ra}$ & $\mathrm{T}$ & $P$ \\
\hline & $\mathrm{N}$ & $\mu \mathrm{m}$ & ${ }^{\circ} \mathrm{C}$ & KW & $\mathrm{N}$ & $\mu \mathrm{m}$ & ${ }^{\circ} \mathrm{C}$ & KW \\
\hline 1 & 296.52 & 2.13 & 62.12 & 0.17 & 328.49 & 2.24 & 64.33 & 0.19 \\
\hline 2 & 365.85 & 2.31 & 72.52 & 0.21 & 390.24 & 2.35 & 76.81 & 0.23 \\
\hline 3 & 412.23 & 2.51 & 81.54 & 0.24 & 433.59 & 2.52 & 85.19 & 0.25 \\
\hline 4 & 296.06 & 2.62 & 75.43 & 0.17 & 318.78 & 2.68 & 79.12 & 0.18 \\
\hline 5 & 359.91 & 2.79 & 85.43 & 0.21 & 381.11 & 2.79 & 89.91 & 0.21 \\
\hline 6 & 400.80 & 2.98 & 94.06 & 0.23 & 425.03 & 2.96 & 96.61 & 0.24 \\
\hline 7 & 307.62 & 3.01 & 85.22 & 0.18 & 331.44 & 3.11 & 90.51 & 0.19 \\
\hline 8 & 365.99 & 3.18 & 94.82 & 0.21 & 394.34 & 3.22 & 99.61 & 0.23 \\
\hline 9 & 401.40 & 3.36 & 103.05 & 0.23 & 438.84 & 3.39 & 104.62 & 0.25 \\
\hline 10 & 285.35 & 1.45 & 55.27 & 0.25 & 316.18 & 1.52 & 59.92 & 0.28 \\
\hline 11 & 353.31 & 1.63 & 68.21 & 0.31 & 378.08 & 1.63 & 76.29 & 0.33 \\
\hline 12 & 398.31 & 1.83 & 79.77 & 0.35 & 421.58 & 1.79 & 88.58 & 0.37 \\
\hline 13 & 287.07 & 1.90 & 67.32 & 0.25 & 310.53 & 1.92 & 73.90 & 0.27 \\
\hline 14 & 349.54 & 2.07 & 79.86 & 0.31 & 373.01 & 2.03 & 88.59 & 0.33 \\
\hline 15 & 389.06 & 2.26 & 91.02 & 0.34 & 417.08 & 2.19 & 99.18 & 0.37 \\
\hline 16 & 300.80 & 2.26 & 75.84 & 0.27 & 327.24 & 2.32 & 84.48 & 0.29 \\
\hline 17 & 357.79 & 2.42 & 87.98 & 0.32 & 390.29 & 2.43 & 97.48 & 0.34 \\
\hline 18 & 391.83 & 2.60 & 98.75 & 0.35 & 434.94 & 2.59 & 106.39 & 0.39 \\
\hline 19 & 278.55 & 1.53 & 53.19 & 0.38 & 307.25 & 1.54 & 54.57 & 0.41 \\
\hline 20 & 344.53 & 1.71 & 69.75 & 0.46 & 369.37 & 1.64 & 76.51 & 0.49 \\
\hline 21 & 387.57 & 1.91 & 84.93 & 0.52 & 413.08 & 1.79 & 94.36 & 0.55 \\
\hline 22 & 283.36 & 1.93 & 63.44 & 0.38 & 307.39 & 1.89 & 67.40 & 0.41 \\
\hline 23 & 343.87 & 2.10 & 79.60 & 0.46 & 370.08 & 1.99 & 87.65 & 0.49 \\
\hline 24 & 381.42 & 2.28 & 94.38 & 0.51 & 414.37 & 2.14 & 103.81 & 0.55 \\
\hline 25 & 300.20 & 2.23 & 70.15 & 0.40 & 329.89 & 2.23 & 76.82 & 0.43 \\
\hline 26 & 355.22 & 2.39 & 85.92 & 0.47 & 393.15 & 2.33 & 95.38 & 0.51 \\
\hline 27 & 387.29 & 2.57 & 100.31 & 0.52 & 438.01 & 2.48 & 109.85 & 0.58 \\
\hline
\end{tabular}

Table 5.Observations under the Application of Coconut and Groundnut Oil (MQL Environment)

\begin{tabular}{|c|c|c|c|c|c|c|c|c|}
\hline \multirow{2}{*}{$\begin{array}{c}\text { Run } \\
\text { No. }\end{array}$} & \multicolumn{4}{|c|}{ MQL-coconut } & \multicolumn{4}{|c|}{ MQL-groundnut } \\
\cline { 2 - 8 } & Fc & Ra & T & P & FC & Ra & T & P \\
\hline
\end{tabular}




\begin{tabular}{|c|c|c|c|c|c|c|c|c|} 
& $\mathrm{N}$ & $\mu \mathrm{m}$ & ${ }^{\circ} \mathrm{C}$ & $\mathrm{KW}$ & $\mathrm{N}$ & $\mu \mathrm{m}$ & ${ }^{\circ} \mathrm{C}$ & $\mathrm{KW}$ \\
\hline 1 & 354.71 & 2.76 & 75.08 & 0.20 & 368.62 & 2.24 & 69.78 & 0.21 \\
\hline 2 & 416.64 & 2.87 & 84.92 & 0.24 & 427.07 & 2.35 & 80.70 & 0.25 \\
\hline 3 & 462.82 & 2.95 & 94.95 & 0.26 & 473.53 & 2.52 & 90.83 & 0.27 \\
\hline 4 & 344.19 & 3.09 & 90.71 & 0.19 & 360.36 & 2.68 & 84.05 & 0.20 \\
\hline 5 & 405.39 & 3.20 & 100.12 & 0.23 & 420.74 & 2.79 & 94.96 & 0.24 \\
\hline 6 & 450.84 & 3.27 & 109.71 & 0.25 & 469.11 & 2.96 & 105.06 & 0.27 \\
\hline 7 & 358.04 & 3.42 & 103.51 & 0.20 & 368.60 & 3.11 & 96.12 & 0.21 \\
\hline 8 & 418.52 & 3.51 & 112.49 & 0.24 & 430.90 & 3.22 & 107.01 & 0.25 \\
\hline 9 & 463.25 & 3.58 & 121.66 & 0.27 & 481.20 & 3.39 & 117.10 & 0.28 \\
\hline 10 & 341.36 & 1.95 & 69.20 & 0.30 & 358.85 & 1.52 & 63.85 & 0.32 \\
\hline 11 & 403.85 & 2.09 & 82.54 & 0.35 & 415.81 & 1.63 & 78.18 & 0.37 \\
\hline 12 & 450.59 & 2.19 & 96.07 & 0.40 & 460.77 & 1.79 & 91.72 & 0.41 \\
\hline 13 & 334.63 & 2.29 & 82.29 & 0.29 & 355.05 & 1.92 & 76.23 & 0.31 \\
\hline 14 & 396.40 & 2.41 & 95.21 & 0.35 & 413.94 & 2.03 & 90.55 & 0.36 \\
\hline 15 & 442.41 & 2.51 & 108.30 & 0.39 & 460.82 & 2.19 & 104.07 & 0.41 \\
\hline 16 & 352.28 & 2.62 & 92.57 & 0.31 & 367.76 & 2.32 & 86.41 & 0.33 \\
\hline 17 & 413.32 & 2.73 & 105.05 & 0.37 & 428.57 & 2.43 & 100.71 & 0.38 \\
\hline 18 & 458.61 & 2.81 & 117.71 & 0.41 & 477.38 & 2.59 & 114.21 & 0.43 \\
\hline 19 & 333.59 & 1.72 & 65.89 & 0.44 & 355.59 & 1.54 & 60.73 & 0.48 \\
\hline 20 & 396.88 & 1.88 & 84.23 & 0.52 & 410.42 & 1.64 & 79.94 & 0.54 \\
\hline 21 & 444.42 & 2.01 & 102.75 & 0.59 & 453.26 & 1.79 & 98.34 & 0.61 \\
\hline 22 & 332.27 & 2.05 & 75.37 & 0.44 & 358.16 & 1.89 & 70.41 & 0.48 \\
\hline 23 & 394.84 & 2.21 & 93.27 & 0.52 & 414.93 & 1.99 & 89.60 & 0.55 \\
\hline 24 & 441.66 & 2.33 & 111.36 & 0.59 & 459.69 & 2.14 & 107.99 & 0.62 \\
\hline 25 & 355.33 & 2.38 & 82.02 & 0.47 & 377.25 & 2.23 & 77.89 & 0.50 \\
\hline 26 & 417.17 & 2.52 & 99.50 & 0.55 & 435.93 & 2.33 & 97.06 & 0.58 \\
\hline 27 & 463.26 & 2.64 & 117.15 & 0.61 & 482.62 & 2.48 & 115.43 & 0.64 \\
\hline
\end{tabular}

\subsection{Performance evaluation of cutting force}

This subsection describes the performance evaluation of cutting force under consideration.

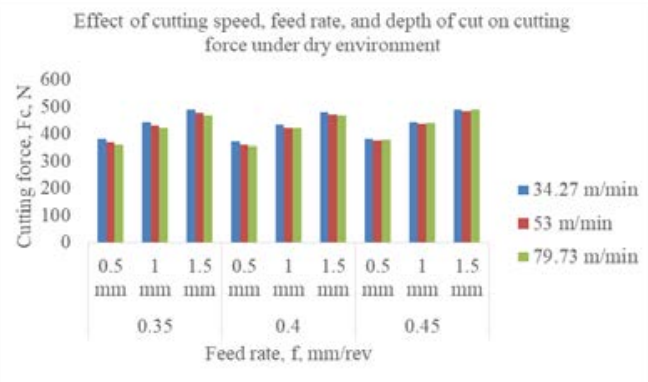

(a) Dry environment
Effect of cutting speed, feed rate, and depth of cut on curting force under flood-blasocut environment

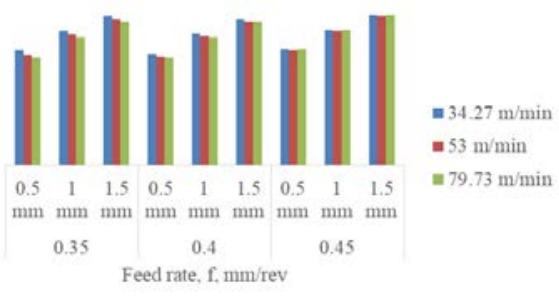

(b) Flood lubrication 


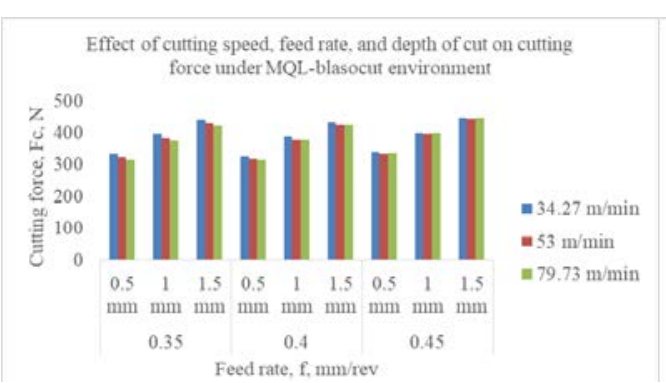

(c) MQL-blasocut

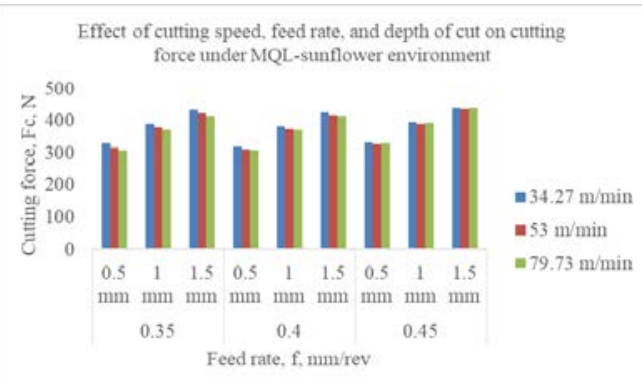

(e) MQL-sunflower

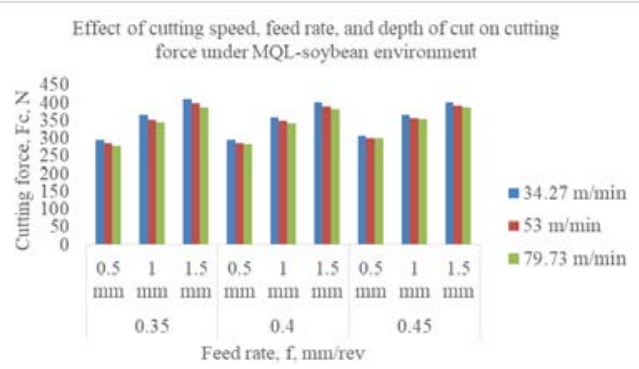

(d) MQL-soybean

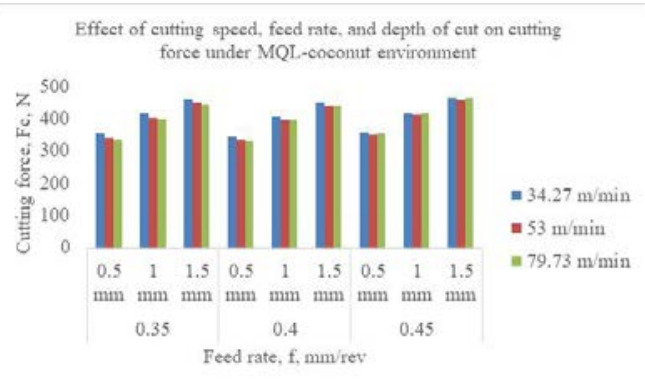

(f) MQL-coconut

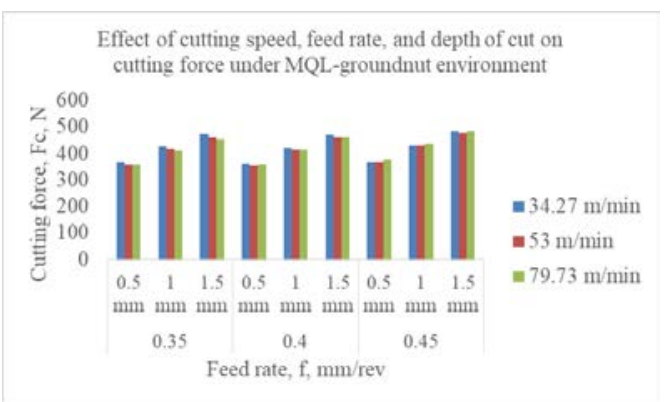

(g) MQL-groundnut

Figure2.Variation of cutting force at different cutting conditions

Figure 2 shows the effect of varying cutting conditions and oil applications on cutting forceunder the different machining conditions. It is clear to note the reduction in cutting forces under vegetable oil application. Comparing vegetable oils with mineral-based oil; gives compatible results. Maximum cutting force is observed for the dry environment as compared to flood and MQL environments. The better performance of the MQL environment is attributed to the high-velocity coolant jet resulting in lower adhesion thereof. MQL shows approximately $5-10 \%$ and $10-15 \%$ reduction in cutting forces as compared to flood and dry cutting environments respectively. MQL with soybean oil gives superior results in overall comparison, which is attributed to more viscosity, less evaporation leading to better cooling of the tool-tip as compared to other oils and cutting environments [32]. Soybean oil application shows a 6-7\% reduction in cutting forces compared to sunflower oil.

\subsection{Performance evaluation of surface roughness}

The performance evaluation of surface roughness is presented here.
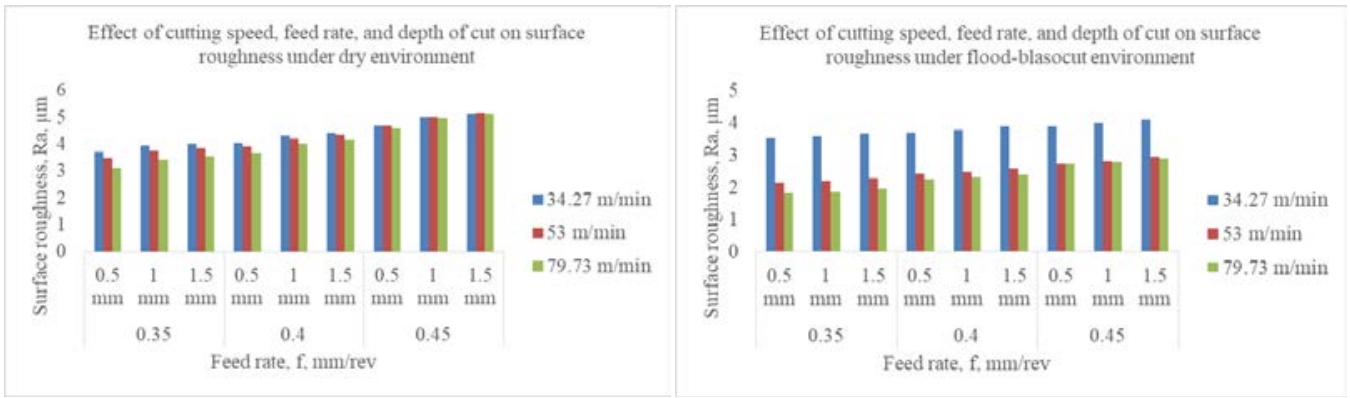

(a) Dry environment

(b) Flood lubrication 


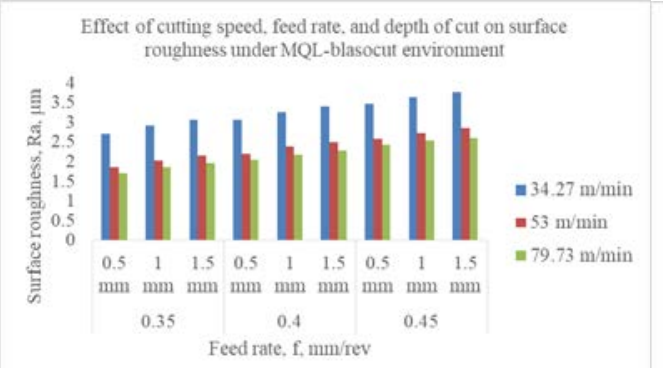

(c) MQL-blasocut

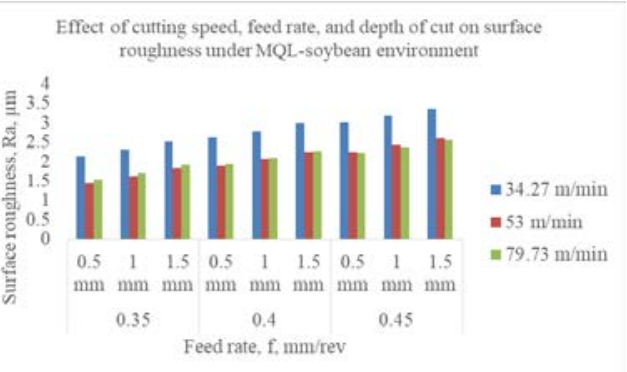

(d) MQL-soybean

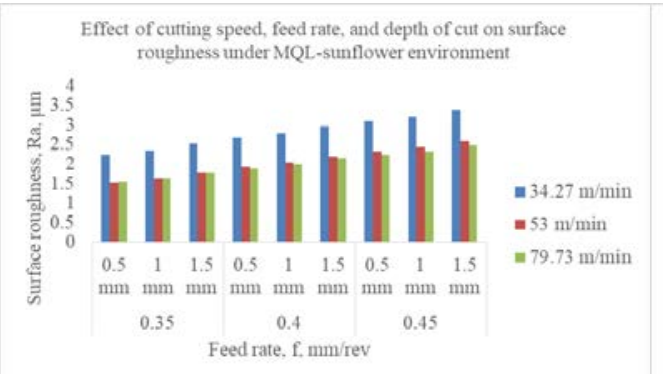

(e) MQL-sunflower

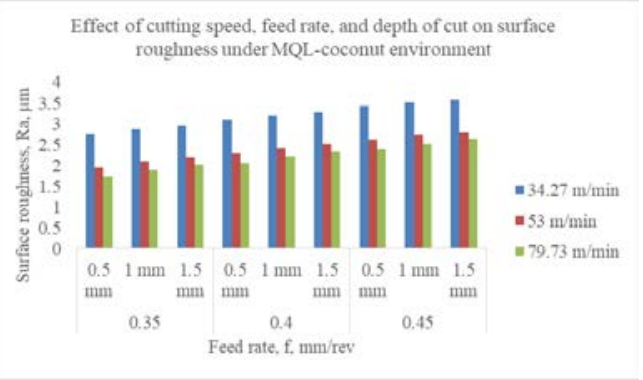

(f) MQL-coconut

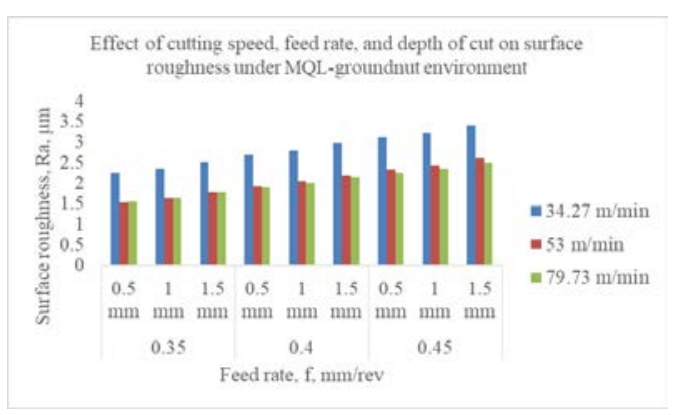

(g) MQL-groundnut

Figure3.Variation of surface roughness at different cutting conditions

Figure 3 shows the effect of varying cutting conditions and oil applications on surfaceroughness under the different machining conditions. As expected, the MQL environment shows superior results over the flood and dry environment. There is an average $8 \%$ and 38\% reduction in surface roughness under MQL conditions over the flood and dry cutting environment respectively. The soybean oil with its high molecular weight and dipolar molecular action resulting in lower cutting forces, better lubrication, and hence the lower toll wear which ultimately leads to lesser tool wear by the virtue of a decrease in temperature. This is overall improves the surface quality of the material under consideration. Comparing the vegetable oils; soybean oil shows improved surface quality by $4 \%, 8 \%$, and $15 \%$ over the sunflower, groundnut, and coconut oil respectively. The reduction in surface roughness at the higher cutting speed is attributed to a decrease in chip-tool contact length.

\subsection{Performance evaluation of temperature}

The performance evaluation of temperature is presented here.

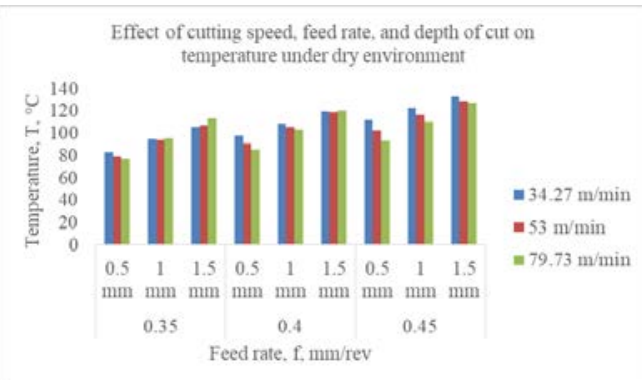

(a) Dry environment

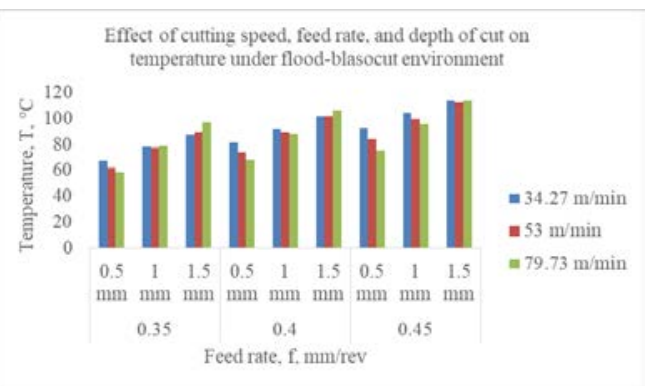

(b) Flood lubrication 


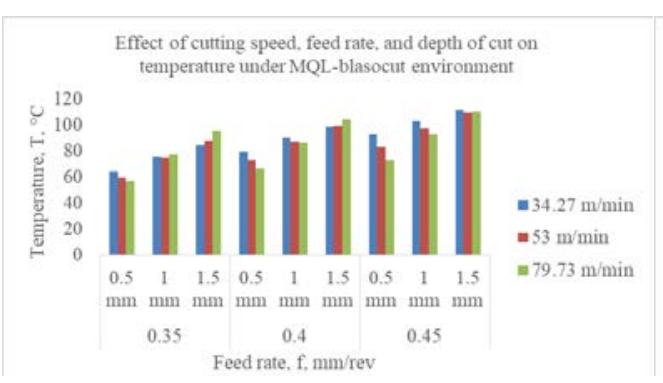

(c) MQL-blasocut

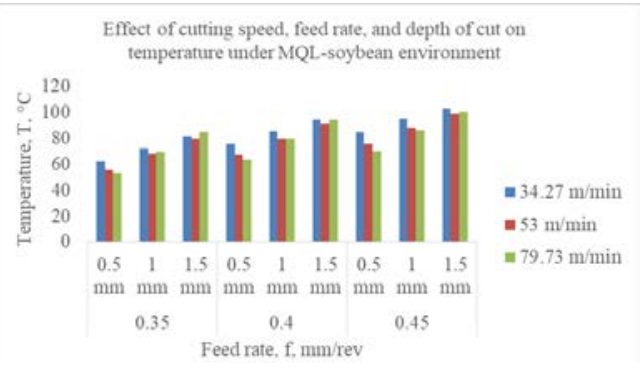

(d) MQL-soybean

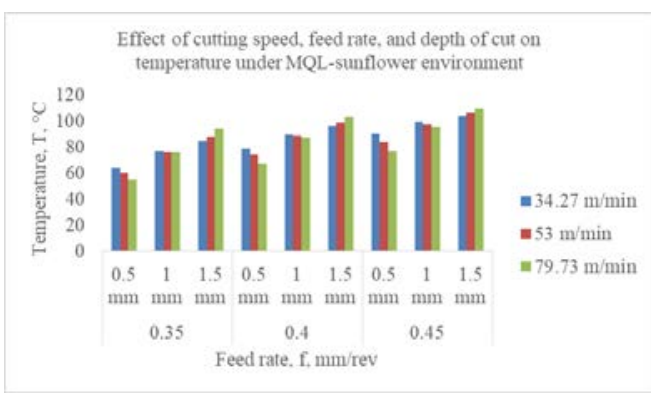

(e) MQL-sunflower

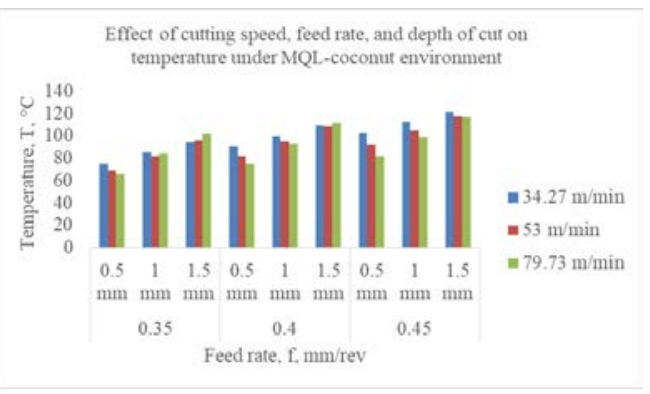

(f) MQL-coconut

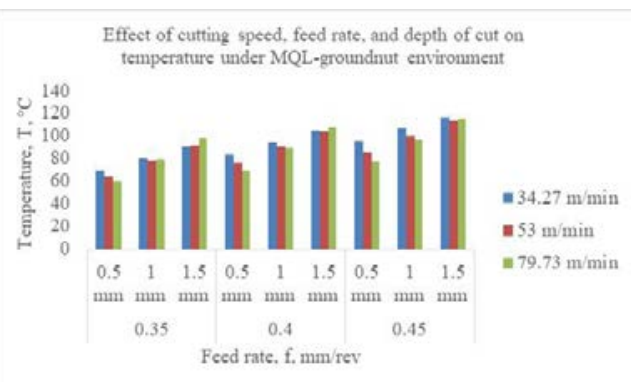

(g) MQL-groundnut

Figure4.Variation of temperature at different cutting conditions

Figure 4 shows the variation of temperature concerning the different cutting conditions and oils. As observed, the increase in depth of cut and feed rate increases the temperature, which is attributed to more friction between the tool and workpiece. MQL environment is better over flood and dry environment to reduce the temperature attributed to heat dissipation by the virtue of air and coolant mixture at high velocity and pressure. MQL helps in reducing the temperature by $10-20 \%$ and $2-5 \%$ as compared to dry and flood environments respectively. Vegetable oils possess high viscosity and do offers better lubrication and better thermal conductivity than mineral oils. The viscosity index of soybean oil is higher than mineral oils and other vegetable oils which gives stability at a higher temperature [33]. Soybean oil shows around a 7\% reduction in temperature comparing the sunflower oil.

\subsection{Performance evaluation of power consumption}

This subsection describes the performance evaluation for power consumption under consideration.

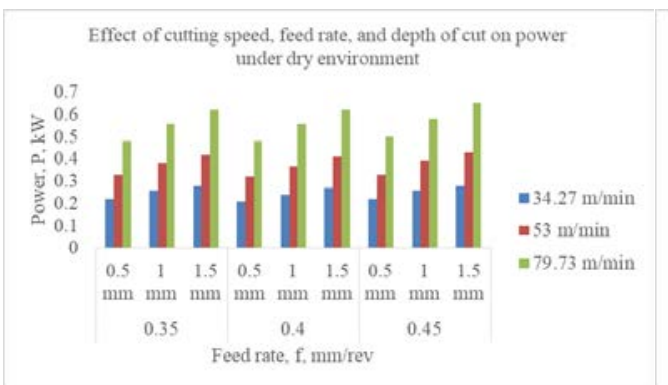

(a) Dry environment

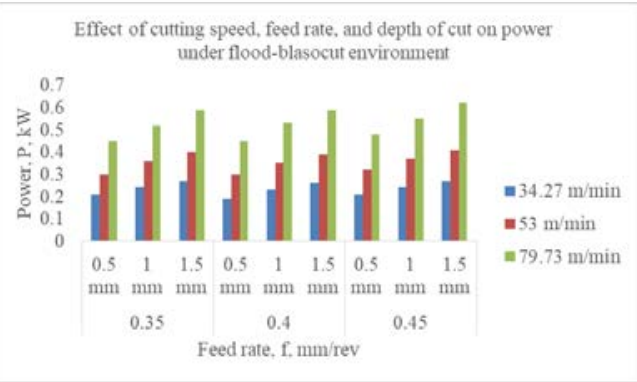

(b) Flood lubrication 


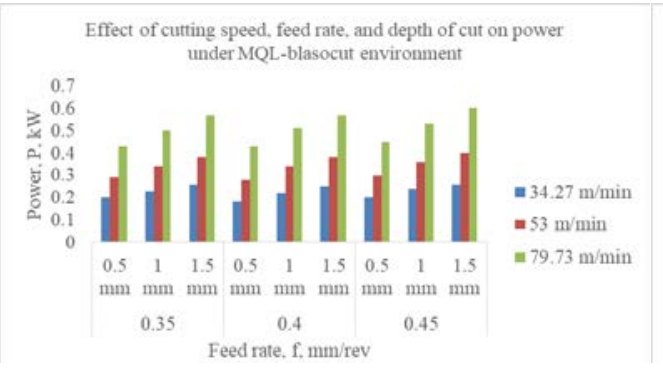

(c) MQL-blasocut

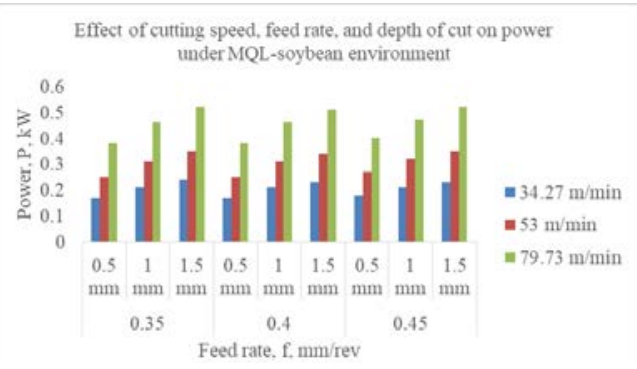

(d) MQL-soybean

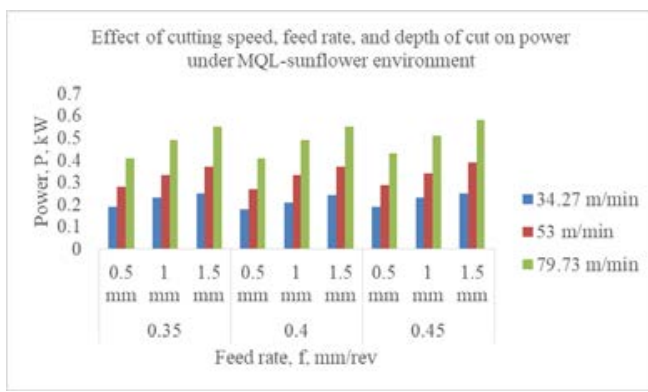

(e) MQL-sunflower

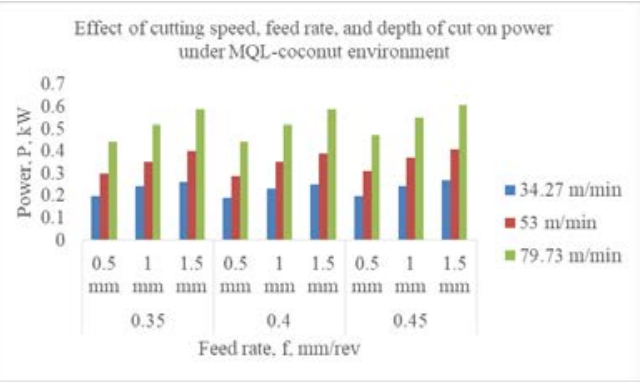

(f) MQL-coconut

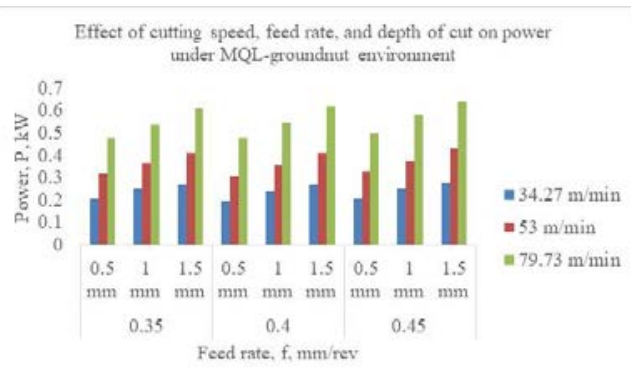

(g) MQL-groundnut

Figure5.Variation of power consumption at different cutting conditions

Figure 5 represents the variation of power consumption at different cutting conditions underconsideration. As speed increases, demand for driving power also increases which results in maximum power consumption. The depth of cut and feed rate is proportionate to the power consumption which is attributed to more frictional resistance at the tool-work interface. Dry cutting incurred maximum power consumption as $5-10 \%$ more than flood cutting; whereas, MQL helps in reducing power consumption approximately by $10 \%$ overall. Soybean oil performs better comparatively against all the conditions and shows about $9 \%$ reduced power consumption as compared to mineral-based blasocut oil.

\section{Conclusions}

The present research work is carried out to evaluate the performance of various vegetable-basedcutting fluids under varying cutting environments concerning the measuring parameters; cutting force, surface roughness, temperature, and power consumption. Based on the comprehensive experimental investigation following conclusions and remarks could be drawn:

- Minimum quantity lubrication shows improved characteristics in all aspects. The cutting forces, temperature, surface roughness, and power consumption found reduced under the application of MQL as compared to the dry and flood lubrication technique. MQL is also useful in the consumption of the cutting fluid

- The vegetable oils under MQL shows significant performance improvement than that of blasocut oil.

- Soybean oil shows the lowest cutting forces as compared to blasocut, by $9 \% /$

- Under the applic ation of soybean oil, the surface roughness was noted to be 
reduced by $4 \%, 8 \%, 15 \%$, and $16 \%$ as compared to sunflower, groundnut, blasocut, and coconut oil respectively.

- The soybean oil is also reported for the lower temperature as compared to blasocut, sunflower, groundnut, and coconut oil by 3\%, 7\%, 12\%, and $16 \%$ respectively.

- Power consumption under soybean oil application is reported to be lower as compared to sunflower, blasocut, coconut, and groundnut oil by $7 \%, 9 \%, 13 \%$, and $19 \%$ respectively.

- The overall observations under all machining environments show that the depth of cut shows a significant effect on cutting forces, feed rate and depth of cut are found to be influential parameters affecting the temperature, and cutting speed are noted to be an influential parameter for the power consumption.

- The combined effect of cooling and lubrication under the application of MQL assisted vegetable oils has shown significant performance comparatively over the other environments. The outstanding performance of the soybean oil is attributed to its higher molecular weight resulting in fewer evaporation losses, and its highest unsaturated fatty acid content resulting in excellent lubrication properties comparatively.

- The soybean oil is less costly as well and based on the investigations it is proposed that the application of soybean oil under the MQL environment would be an excellent substitute for the mineral-based cutting fluids.

\section{References}

[1] M. Sokovic and K. Mijanovic, "Ecological aspects of the cutting fluids and its influence on the quantifiable parameters of the cutting processes,” Journal of Materials Processing Technology, Vol. 109, (2001), pp. 181-189.

[2] D. P. Adler, W. W.S. Hii, D, D. J. Michalek, and J. W. Sutherlamd, "Examining the role of cutting fluids in machining and efforts to address associated environmental/health concerns," Machining Science and Technology, Vol. 10-1, (2006), pp. 23-58.

[3] Bennett EO and Bennett DL, "Minimizing human exposure to chemicals in metalworking fluids", Lubrication Engineering, Vol.43-3, (1987), pp. 167-175.

[4] L. K. Low, J. R. Meeks, C.R. Mackere, "Health effects of alkyl benzenes II. Xylenes”, Toxicology and Industry Health, Vol. 5-1, (1989), pp. 85-105.

[5] Simpson AT, Stear M, Groves JA, Piney M, Bradley SD, Stagg S, Crook B, “Occupational exposure to metalworking fluid mist and sump fluid contaminants”, Annals of Occup. Hyg., Vol. 47, (2003), pp. 1730.

[6] Klocke F. and Eisenblatter G, "Dry cutting”, Annals of the CIRP, Vol. 46-2, (1997), pp. 519-526.

[7] Dixit U.S, Sarma D.K, Davin J.P, “Environmentally friendly machining”, ISBN 978-1-4614-2308-9, Springer-Verlag New York, (2012), pp.1-100.

[8] P.S. Sreejith and B.K.A. Ngoi, "Dry machining-machining of the future”, Journal of Materials Processing Technology, Vol. 101,1-3, (2000), pp 289-293.

[9] T.F. MaClure, R. Adams, M.D. Gugger, "Comparison of flood vsmicrolubrication on machining performance”, http://www.unist.com/techsolve.html., (2001), pp. 4-5.

[10] N.R. Dhar, M. Kamruzzamman, M. Ahmed, "Effect of minimum quantity lubrication (MQL) on tool wear and surface roughness in turning AISI-4340 steel”, J. Mater. Process. Technol. Vol. 172-2, (2006), pp. 299-304.

[11] N. R. Dhar, M. T. Ahmed, S. Islam, "An experiment investigation on effect of minimum quantity lubrication in machining AISI 1040 steel”, International Journal of Machine Tools \& Manufacture, Vol. 47, (2007), pp. 748-753.

[12] AhmadrezHosseiniTazehkandi, "On the feasibility of a reduction in cutting fluid consumption via spray of biodegradable vegetable oil with compressed air in machining of Inconel 706" Journal Of Cleaner Production, Vol. 104, (2015), pp.422-435.

[13] A.S. Varadarajan and P.K. Philip, "Investigations on hard turning with minimal cutting fluid application and its comparison with dry and wet turning”, International Journal of Machine Tools and Manufacture, Vol. 42-2, (2002), pp. 193-200.

[14] C. Ju, L. P. Keranen, K. R. Haapala, D. J. Michalek, J. W. Suthrland, “Issues associated with MQL 
implementation: effect on peripheral milling process performance and impact on machining economics”. Proc. of ASME Manufacturing Engineering Division, Orlando, Florida, 2005, (IMECE2005-79259) IMECE Paper -79259, (2005), pp.3-12.

[15] Attanasio , M. Gelfi, C. Giardini, C. Remino "Minimal quantity lubrication in turning effect on tool wear” Wear, Vol. 260, (2006), pp. 333-338.

[16] Y.S. Liao, H.M. Lin, Y.C. Chen, "Feasibility study of the minimum quantity lubrication in high-speed end milling of NAK80 hardened steel by coated carbide tool”, International Journal of Machine Tools \&Manufacture, Vol-47, (2007), pp. 1667-1676.

[17] M.C.S. Alves, E.C, Bianchi, P.R. Aguiar, R.C. Canarim, "Influence of optimized lubrication cooling and MQL on the cutting force, on the geometric quality of the surfaces and on the micro structural integrity of hardened steel part”, RevistaMateria, (Rio the Janeiro) , Vol. 16-3, (2011),pp.754-766.

[18] Hadad, M. and Sadeghi, B. "Minimum quantity lubrication-turning of AISI 4140 steel alloy”, Journal of Cleaner Production, Vol.54, (2013), pp.332-343.

[19] Kedare S.B, Borse, D.R., Shahane P. T., "Effect of minimum quantity lubrication on surface roughness of mild steel of 15HRC on universal milling machine”, Procedia Materials Science, ICMPC 2014, Vol.6, (2014), pp. 150-153.

[20] M.M.A. Khan., M.A.H. Mithu, N.R. Dhar, "Effects of minimum quantity lubrication on turning AISI 9310 alloy steel using vegetable oil-based cutting fluid”, Journal of Materials Processing Technology, Vol. 209, (2009), pp. 5573-5583.

[21] E. Kuram, B. Ozcelik, E. Demirbas, E. Sik, I. N. Tansel, "Evaluation of new vegetable-based cutting fluids on thrust force and surface roughness in drilling of AISI 304 using taguchi method", Materials and Manufacturing Processes, Vol. 26, (2011), pp.1136-1146.

[22] Khan M.M.A. and Dhar N.R, "Performance evaluation of minimum quantity lubrication by vegetable oil in terms of cutting force, cutting zone temperature, tool wear, job dimension and surface finish in turning AISI-1060 steel”, Journal of Zhejiang University, Science, Vol. 7-11, (2006), pp. 1790-1799.

[23] M A. Islam and N.R. Dhar, "Effects of Minimum Quantity Lubrication by vegetable oil based cutting fluid on temperature, chip morphology and surface finish in grinding AISI 1060 steel”, Proceedings of the 4th BSME-ASME International Conference on Thermal Engineering, 27-29 December (2008), Dhaka, Bangladesh, pp.861-866.

[24] Gunjal S. U. and Patil N. G, "Experimental investigations into turning of hardened AISI 4340 steel using vegetable based cutting fluids under minimum quantity lubrication.” ProcediaManufacturing, 20, (2018), pp.18-23.

[25] S. U. Gunjal, S. B. Sanap, N. G. Patil, "Role of cutting fluids under minimum quantity lubrication: An experimental investigation of chip thickness”, Materials Today: Proceedings, Volume 28-2, (2020), pp 1101-1105.

[26] Shrikant U. Gunjal and Sudarshan B. Sanap, "Experimental investigations and regression analysis based mathematical modelling for tool life under minimum quantity lubrication”, International Journal of Mechanical and Production Engineering Research and Development, Vol. 10, (2020), pp 911-920.

[27] Y. M. Shashidhara and S. R. Jayaram, "Experimental determination of cutting power for turning and material removal rate for drilling of AA 6061-T6 using vegetable oils as cutting fluid”, Advances in Tribology, Hindawi Publishing Corporation, Vol. 2013, (2013) Article ID 362931, pp.1-7.

[28] Manoj Kumar, JeewanSarda, AmitavaGhosh, "Potential of vegetable oils as micro lubrication/cooling medium for SQL grinding”, 5th International \& 26th All India Manufacturing Technology, Design and Research Conference (AIMTDR 2014) December 12th-14 ${ }^{\text {th }}$, IIT Guwahati, Assam, India 387, (2014), pp.1-5.

[29] Jagdeep Sharma and Balwinder Singh Sidhu, "Investigation of effect of dry and near dry machining on AISI D2 steel using vegetable oil”, Journal of Cleaner Production, Vol. 66-03, (2014), pp. 619-623.

[30] Mohamed HindawiSaadElmunafi, D. Kurniawan, M. Y. Noordin, "Use of castor oil as cutting fluid on machining of hardened stainless steel with minimum quantity lubrication”, Procedia CIRP, Vol. 26, (2015), pp. 408-411.

[31] Shrikant U. Gunjal and Sudarshan B. Sanap, “A bibliometric analysis of minimum quantity lubrication as a sustainable approach”, Library Philosophy and Practices, (2020), 4772.

[32] Gurpreet Singh, Sehijpal Singh, Manjot Singh, Ajay Kumar, "Experimental investigations of vegetable \& mineral oil performance during machining of EN-31 steel with minimum quantity lubrication”, International Journal of Research in Engineering and Technology, Vol. 02-6, (2013), pp.1030-1037.

[33] O.O. Fasina and Z. Colley, "Viscosity and specific heat of vegetable oils as a function of temperature", International journal of food properties, Vol. 11, (2008), pp 738-74. 
